\title{
Dual-interface gratings for broadband absorption enhancement in thin-film solar cells
}

\author{
Aimi Abass, ${ }^{1, *}$ Khai Q. Le, ${ }^{2}$ Andrea Alù, ${ }^{2}$ Marc Burgelman, ${ }^{1}$ and Bjorn Maes ${ }^{3,4}$ \\ ${ }^{1}$ Department of Electronics and Information Systems, Solar Cells Group, Ghent University, B-9000 Ghent, Belgium \\ ${ }^{2}$ Department of Electrical and Computer Engineering, Metamaterials and Plasmonics Research Group, \\ The University of Texas at Austin, Austin, Texas, USA \\ ${ }^{3}$ Department of Information Technology, Photonics Research Group, Ghent University-imec, B-9000 Ghent, Belgium \\ ${ }^{4}$ Department of Physics, Micro- and Nanophotonic Materials Group, University of Mons, B-7000 Mons, Belgium
}

(Received 9 February 2012; published 30 March 2012)

\begin{abstract}
We numerically study complex dual-interface grating systems to enhance absorption efficiency in thin-film silicon solar cells. We combine a plasmonic grating at the back side of the solar cell with a dielectric grating at the front side of the cell. We show a proof of principle, with one-dimensional gratings, that the distinctly different nature of the gratings can provide complementary enhancement mechanisms, which we further exploit by tailoring the specific periodicities, and by introducing blazing. Having different periods at specific interfaces allows for more efficient diffraction into both plasmonic and dielectric guided modes. In addition, grating specific blazing exposes extra modes to normal incident light through symmetry breaking. Multiple optimization routes are possible depending on the choice of photonic phenomena.
\end{abstract}

DOI: 10.1103/PhysRevB.85.115449

PACS number(s): 42.25.Bs, 42.25.Fx, 42.30.Kq

\section{INTRODUCTION}

To meet growing energy demands in a sustainable way, significant research has been focused on developing economical thin-film solar cells. Without sophisticated light-trapping techniques, these cells tend to have low light-absorption efficiency due to their limited active layer thickness. ${ }^{1}$ The necessity for absorption enhancement, along with the development of fabrication technology at the nanoscale, has sparked large interest in light-trapping nanostructures. ${ }^{2-6}$ Several nanostructured materials have been proposed, both periodic and aperiodic. $^{7-11}$ Various types of periodic gratings can provide significant absorption enhancements through diverse photonic phenomena such as scattering, diffraction, and coupling into waveguide modes or surface plasmons. ${ }^{8-10,12-21}$ The idea of using surface plasmons to enhance thin-film solar cells has raised much interest because of the strong scattering and near-field confinement ${ }^{12,13,22-26}$ typical of these modes. Though they are confined close to the metal surface, absorption can primarily occur in the active layer. ${ }^{5,15,17,22}$

Currently, there is growing interest in using multiple grating structures to enhance absorption, especially in devices combining front and back gratings. ${ }^{13,14,21}$ In some fabrication processes these dual-interface systems may be introduced with no significant difficulty. ${ }^{21}$ Different gratings complement each other by exciting different photonic phenomena that boost absorption in nonoverlapping wavelength ranges, resulting in an overall broad absorption enhancement.

Here, we propose the use of triangular dual-interface grating (DIG) structures with different materials, and numerically study their extensive mode-coupling possibilities. We employ one-dimensional (1D) gratings to keep both the analysis and calculations more easily tractable for understanding the phenomena. In reality, these structures can be extended to be polarization independent via the more conventional twodimensional (2D) gratings. We discuss the benefits of having multiperiod DIG gratings, i.e., a different periodicity for the top and bottom grating. This type of device offers the possibility of enhancing the diffraction coupling to multiple waveguide and surface plasmon polariton (SPP) modes simultaneously, over different wavelength ranges. In addition, we include symmetry-breaking, blazing properties that may excite previously inaccessible modes for normal incident light, further broadening the enhancement range. We focus on structures with semiconductor triangular gratings on top, combined with metal gratings on the back; however, the proposed principles can be adapted to other material combinations.

Section II introduces the employed grating systems and the initial design principles. Section III discusses the starting, symmetric single-periodic DIG structures, which can already provide significant enhancement. Section IV extends the device to multiperiodic structures and explains the operating principles. Section V further tailors the performance by adding blazed, asymmetric features. We summarize and draw conclusions in Sec. VI.

\section{DESIGN STRATEGY}

Our starting design with dual-interface triangular gratings [Fig. 1(a)] uses amorphous $\mathrm{Si}$ (a-Si) as the active layer material. This type of layer and grating structure, with dimensions in the same range as those of the structure in Fig. 1(b), provides a system where guided or Bloch mode engineering can play a significant role in the overall absorption enhancement, as will be discussed in the following sections.

For the front grating (at the air side), we should ensure that a large proportion of the incoming light enters the solar cell. Therefore we add a gradually varying effective index approach by using triangles in the indium tin oxide (ITO) contact. This gradual approach with subwavelength features is useful as an effective antireflection coating with broadband transmission. ${ }^{3,27-29}$ For the metal back grating we also use a triangular geometry, as we previously demonstrated a flexible scattering capability and beneficial field distribution of SPP modes. ${ }^{15}$ Basic design guidelines for the dimensions of the grating structures for placing waveguide and SPP mode resonances are obtained by folded dispersion diagrams. ${ }^{15}$ The fact that the triangular grating geometry also changes the 
(a)

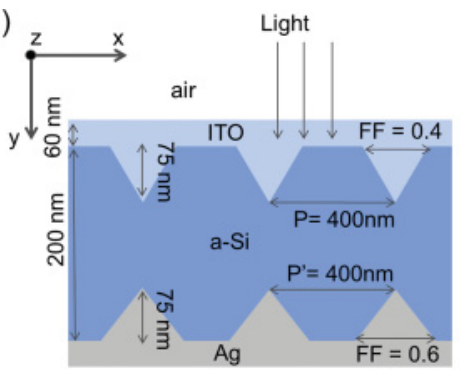

(b)

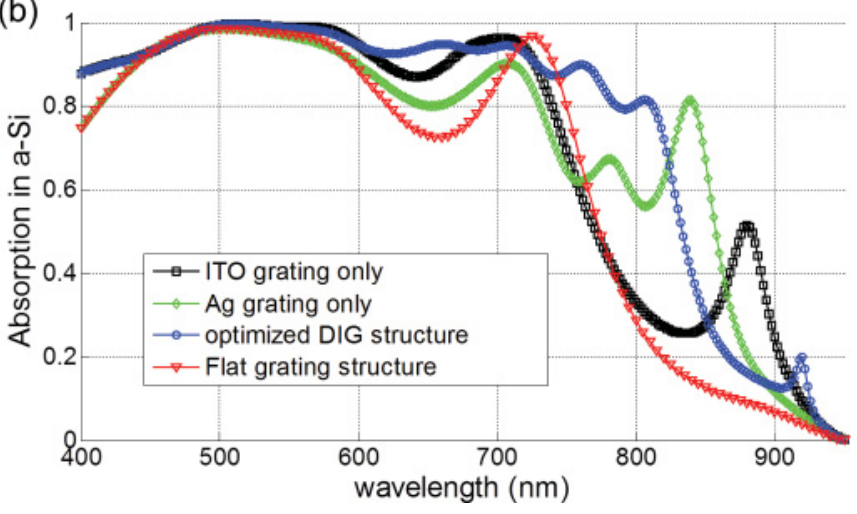

FIG. 1. (Color online) (a) Model of the simulated a-Si grating system. (b) Normal incidence absorption spectrum for flat structure (red triangles), ITO front grating only (black squares), Ag back grating only (green diamonds), and DIG structure (blue circles).

effective layer thicknesses should be taken into account, as it affects the modal dispersion relations.

Here, as we mainly aim to present proof of principles, we focus on 1D grating structures: Full-wave 2D simulations and eigenmodal analyses have been performed using finite element techniques with COMSOL MULTIPHYSICS. One can very often derive three-dimensional structures which respond to arbitrary light polarization in a similar way as the 2D case with transverse magnetic (TM) polarization, if plasmonic effects are exploited correctly. For this reason, we mainly consider TM polarization, in which the magnetic field has one component in the $z$ direction [Fig. 1(a)]. Refractive index data are taken from databases for silver ${ }^{30}$ and for $\mathrm{a}-\mathrm{Si}^{31}{ }^{31}$ To calculate the overall absorption efficiency, we integrate the divergence of the Poynting vector in the active layer and normalize it to the incoming power. We assume perfect quantum efficiency throughout the active layer; therefore absorption everywhere in the active layer is equally important.

\section{SYMMETRIC DUAL-INTERFACE GRATINGS}

In this section, we examine a symmetric and single-periodic DIG structure with optimized triangle heights and grating fill factors (FF); see Fig. 1(a). Figure 1(b) shows the absorption spectra in the a-Si active layer for increasingly complex geometries: flat structure, front and back grating structure only, and for a DIG structure.

Enhancement with the ITO grating only [Fig. 1(b), black square curve] occurs at shorter wavelengths $(<720 \mathrm{~nm})$ and via a resonance peak due to (dielectric) waveguide mode excitation around $880 \mathrm{~nm}$. A profile plot of the absorption and power flow
High
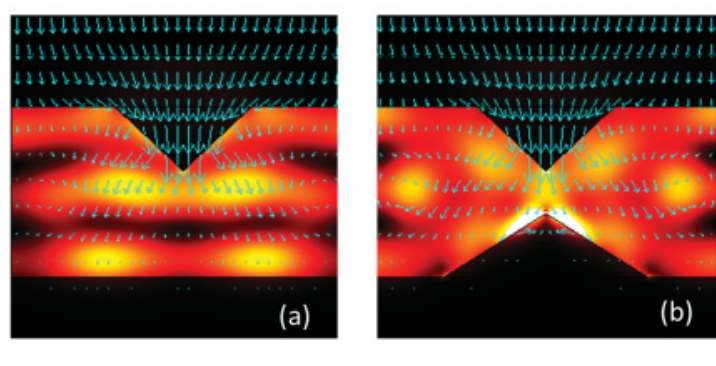

Low

FIG. 2. (Color online) The Poynting vector (arrows) and its divergence (absorption profile) at 660-nm wavelength in the case of (a) ITO grating system only, and (b) DIG system.

at $660 \mathrm{~nm}$ [Fig. 2(a)] reveals that the main mechanism for the enhancement at shorter wavelengths is due to scattering by the ITO triangle features. The ITO triangles focus the incoming light, and subsequently spread it inside the active layer [Fig. 2(a)].

For the structure with Ag back contact only [Fig. 1(b), green diamond curve] the main enhancement occurs at longer wavelengths $(>750 \mathrm{~nm})$, due to the excitation of a waveguide mode at $780 \mathrm{~nm}$ and a plasmonic mode at $838 \mathrm{~nm}$. The wavelength region $<750 \mathrm{~nm}$ is affected minimally by the metal grating structure.

When we combine both grating structures, we obtain a complementary enhancement [Fig. 1(b), blue circle curve]. The scattering power of the front ITO grating structure at shorter wavelengths is still present, as incoming light focusing and spreading into the a-Si layer occurs [Fig. 2(b)]. Additionally, see the blue circle curve in Fig. 1(b), which shows that a combination of dielectric waveguide mode and Fabry-Perot resonance arises at $660 \mathrm{~nm}$, weakly excited together with a plasmonic near-field enhancement near the metal tip [Fig. 2(b)], which together help boost the overall absorption. The magnetic field profile plots for the DIG case (Fig. 3) reveal that the absorption peak at $760 \mathrm{~nm}$ is mainly a waveguide mode (strong field inside the a-Si layer), whereas the peak at $810 \mathrm{~nm}$ corresponds to a plasmonic mode (strong field at the metal-silicon interface). Wavelength shifts of the waveguide and SPP modes are due to changes in the effective thickness and effective index by introducing the metallic and ITO gratings.

In the end, the combined grating structure leads to a better overall enhancement. We calculate an integrated absorption (TM polarization) of the AM1.5G spectrum in the
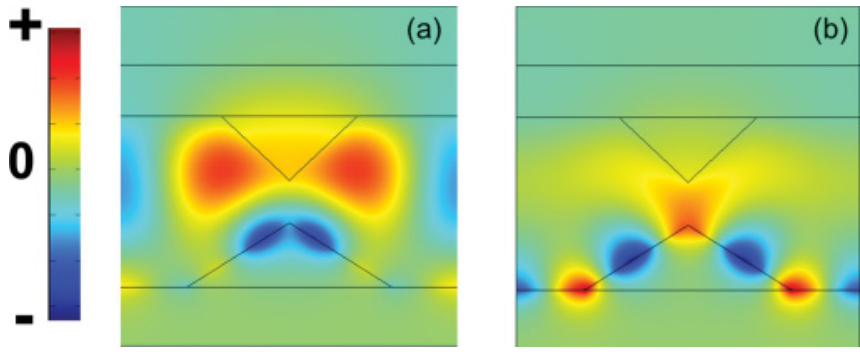

FIG. 3. (Color online) Magnetic field $\left(H_{z}\right)$ profiles (snapshot in time) for the DIG structure at (a) $760 \mathrm{~nm}$ and (b) $810 \mathrm{~nm}$. 
400-950-nm-wavelength region of $83.10 \%$ for the DIG structure and $71.96 \%$ for the flat structure.

\section{DUAL-PERIODIC DUAL-INTERFACE GRATINGS}

For light-trapping purposes it is desirable that the incoming broadband radiation couples to as many (dielectric) waveguide and SPP modes as possible in the solar cell. Essentially, the coupling of incoming light to modes via gratings depends on momentum conservation, the eigen-field profiles of these modes, and the scattering properties of the gratings. ${ }^{32}$ The propagation vector ( $k$ vector) requirement is fulfilled by selecting the proper periodicity regardless of the grating geometry. ${ }^{32,33}$ However, the other two factors strongly influence the coupling efficiency. For example, with symmetric grating systems (such as the one discussed above) there will be modes unexcitable by normal incident plane waves due to their field profiles, both for plasmonic and nonplasmonic modes. These guided modes are "dark modes" and have antisymmetric eigenmode field profiles at $k_{x}=0 .{ }^{15,34}$ The modes with symmetric field profile at $k_{x}=0$ are, on the contrary, "bright modes."

Even for waveguide and SPP modes that are accessible at normal incidence, coupling efficiency may be low due to mismatching between scattering and modal field profiles. In general, there is a trend that lower diffraction orders have a better coupling efficiency than higher-order ones. ${ }^{35}$ Even though access to more modes is possible with larger periodicity, the scattering properties of a normal grating system do not commonly support efficient higher-order diffraction coupling. With higher-order diffraction we mean that Fourier components with momentum $n \frac{2 \pi}{P}$, with $P$ the period and $n>1$, are involved. This higher-order diffraction to guided modes problem (with larger $k_{x}$ ) can be alleviated by having stronger small-periodicity components in the grating system so that their scattering properties can accommodate the amplitude oscillations and shorter wavelengths. Instead of just superimposing different periodicity components on one surface, we propose the usage of different periodicities on both interfaces, ensuring more flexibility in the design. This structure tackles the problem of providing efficient higher-order diffraction coupling to multiple waveguide and SPP modes by effectively lowering their diffraction order while keeping the fundamental order accessible, as will be shown below.

We specifically consider multiperiodic DIG structures in which the back metal grating has a smaller period than the top ITO grating [Fig. 4(c)] for several reasons. First, the plasmonic back grating has a higher scattering cross section as compared to the front ITO grating, and thus affects the scattering properties more. Second, plasmonic modes are localized to the surface of the metal and thus these modes are not sensitive to the top grating. Therefore, in order to improve higher-order diffraction coupling to plasmonic modes (which have a larger $k_{x}$ ), it is necessary to have a smaller periodicity on the back surface. The larger periodicity at the top grating is more suited for dielectric modes, as they are more influenced by that top grating, and they have a smaller $k_{x}$. We focus on dual-periodic DIG systems in which the back grating periodicity is half of the macro periodicity of the whole structure. With this configuration we aim to increase diffraction for second-

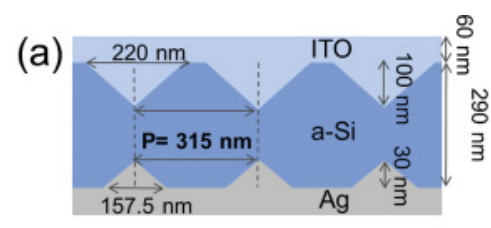

(b)

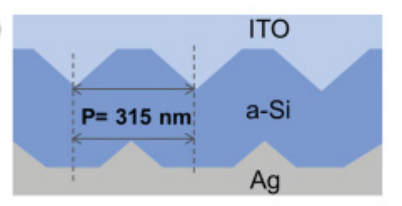

(c)
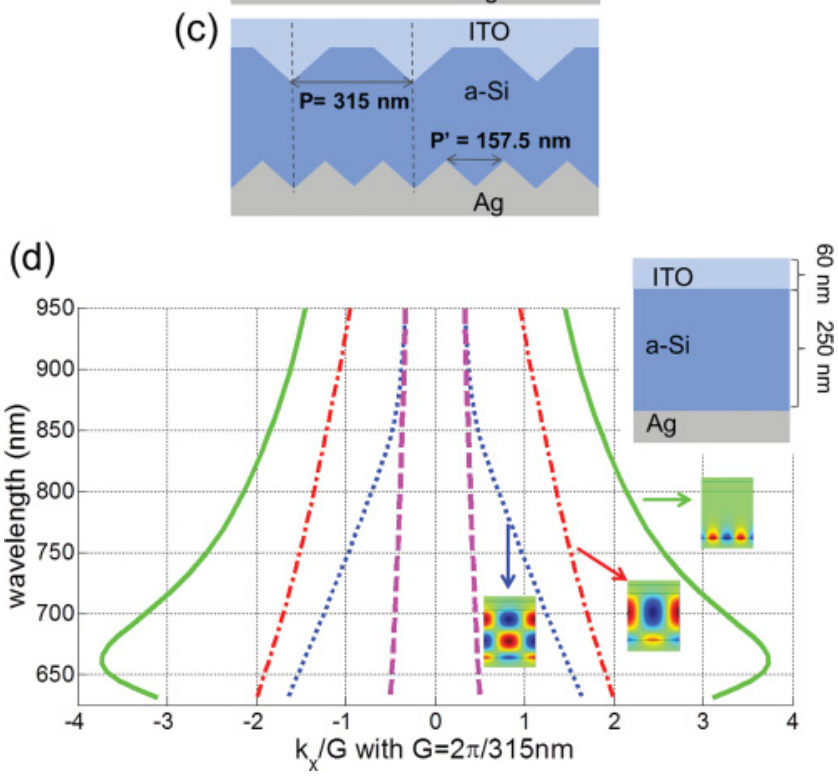

FIG. 4. (Color online) Compared grating structures with $P=$ $315 \mathrm{~nm}$ (a) in phase gratings, (b) $\pi$ phase-shifted gratings, and (c) dual-periodic DIG system with the same macro periodicity $(P=315 \mathrm{~nm})$ and smaller periodicity $\left(P^{\prime}=157.5 \mathrm{~nm}\right)$. (d) Dispersion diagram of waveguide and SPP modes in the indicated flat solar cell structure. Green solid line is for SPP mode, red dash-dot line for first-order waveguide mode, blue dots for second-order waveguide mode, and the pink dashed line indicates the light line in air.

order processes (according to the macro periodicity) while maintaining the efficiency for first-order processes.

We compare systems with the same macro periodicity $(P=$ $315 \mathrm{~nm}$ ); see Figs. 4(a)-4(c). Here, $P$ is chosen in order to have as many first-order diffraction coupling to dielectric waveguide modes as possible, while having higher-order diffraction coupling to SPP modes in the 700-950-nm-wavelength region. The accessible waveguide and SPP modes will still be largely determined by the macro periodicity. Via the dispersion relation of the planar structure [Fig. 4(d) and inset] we can design the gratings to accommodate coupling to the chosen modes. The $k_{x}$ axis in Fig. 4(d) is normalized with respect to $G=\frac{2 \pi}{P}$. The effective thickness of the a-Si layer in the cells in Figs. 4(a)-4(c) is similar to the planar reference thickness.

These structures [Figs. 4(a)-4(c)] support one SPP and two waveguide bright modes in the wavelength region above $625 \mathrm{~nm}$ [Fig. 4(d)]. The peculiar bending of the SPP dispersion is due to the refractive index of a-Si. The crossing points of the dispersion curves with the vertical lines (at integers) indicate the conditions for which the modes can be excited by normal incident light, when we introduce a grating structure 


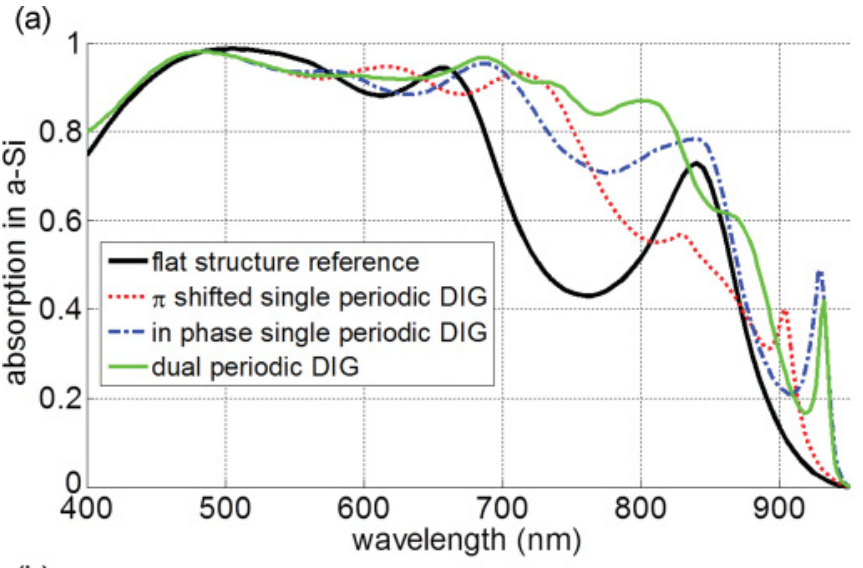

(b)

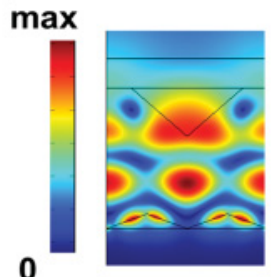

734 nm

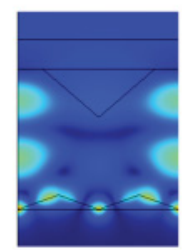

$800 \mathrm{~nm}$

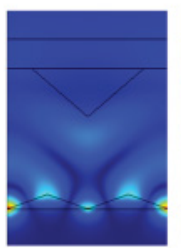

$876 \mathrm{~nm}$

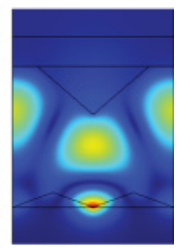

$932 \mathrm{~nm}$

FIG. 5. (Color online) (a) Absorption spectra of DIG systems in Figs. 4(a)-4(c). (b) $\left|H_{z}\right|$ field profiles at the resonances for the dual-periodic structure.

with periodicity $P=315 \mathrm{~nm}$. The integer value of $k_{x} / G$ at these crossings indicates the diffraction order. For $P=315 \mathrm{~nm}$ normal incident light can access the first-order waveguide mode around $930 \mathrm{~nm}$, the second-order waveguide mode around $750 \mathrm{~nm}$, and the SPP mode around $825 \mathrm{~nm}$. Note that the diffraction coupling to the SPP is second order, but for the waveguide modes it is first order.

The absorption spectra for normal incidence are shown in Fig. 5(a). The dual-periodic DIG system [green line in Fig. 5(a)] is able to excite more resonances in a more efficient manner as compared to the single-period systems [blue dashdotted and red dotted lines in Fig. 5(a)].

The magnetic field profiles for the dual-periodicity system [Fig. 5(b)] show the types of excited modes. At the wavelength of $734 \mathrm{~nm}$ we notice a second-order waveguide mode. At 800 and $876 \mathrm{~nm}$, we see a mixed profile arising from the interferences of plasmonic and dielectric modes (they are relatively close and broadband in wavelength). Besides the waveguide and SPP modes, we also expect a Fabry-Pérot resonance around this wavelength range, as can be seen from the flat structure absorption spectrum. Eigenmode calculations (not shown) for structures with gradually decreasing triangle height reveal that the peaks at 800 and $876 \mathrm{~nm}$ stem from a SPP mode and a Fabry-Pérot mode. The profile at $932 \mathrm{~nm}$ is distinctively a firstorder waveguide mode. Thus after accounting for all the resonances, we see that not only do we have boosted second-order diffraction coupling to the SPP mode, but we also maintained strong first-order diffraction coupling to both dielectric modes.

This dual-interface multiperiodicity is especially promising when working with a larger macroperiod, as this leads to more higher-order diffractions in the wavelength range of interest. To show this we compare single- and dual-periodic symmetric

(a)

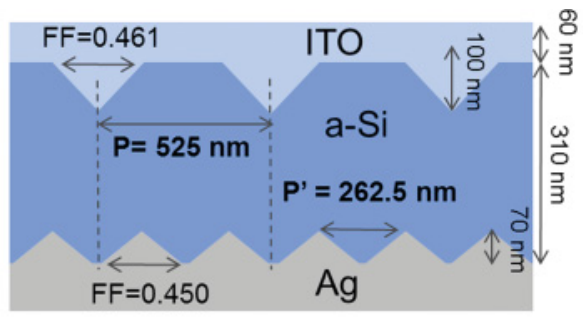

(b)

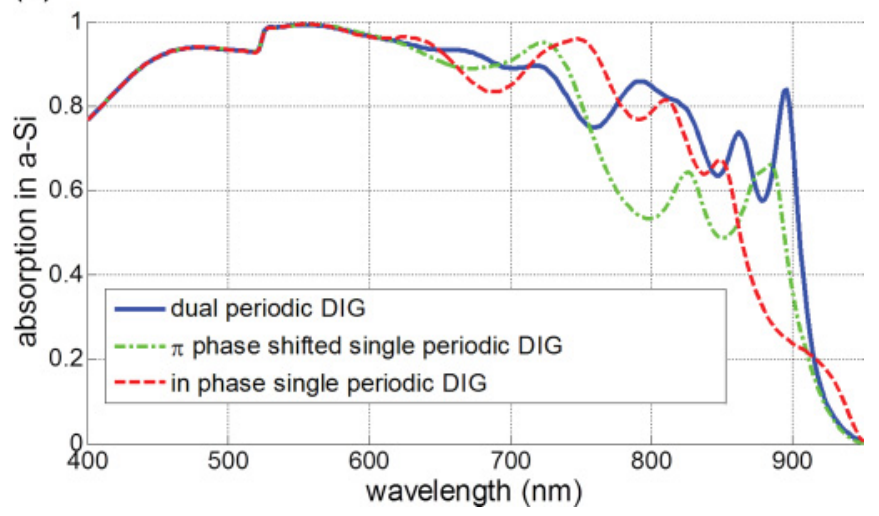

FIG. 6. (Color online) Comparing single and dual periodicity for larger periods. (a) Schematics of the symmetric dual-periodic DIG system with macro periodicity $P=525 \mathrm{~nm}$ and smaller periodicity of $P^{\prime}=262.5 \mathrm{~nm}$. (b) Absorption spectra comparison like that in Fig. 5 for the dual-periodic DIG system in (a) and symmetric single-periodic DIG systems with $525 \mathrm{~nm}$ periodicity that has the same triangle geometries.

systems with a larger macroperiodicity of $525 \mathrm{~nm}$ [Fig. 6(a)]. In Fig. 6(b) we see that the single-periodicity structures can still provide good coupling efficiency for some of the resonances. However, the dual periodicity gives an overall better coupling efficiency and access to more resonances. The field profiles for the resonances at longer wavelengths in Fig. 7 indicate that most of the excited modes arise from higher-order diffraction coupling. The multiple amplitude oscillations in the horizontal direction for the resonance at 792, 826, and $896 \mathrm{~nm}$ indicate that they are excited with higher-order diffraction processes.

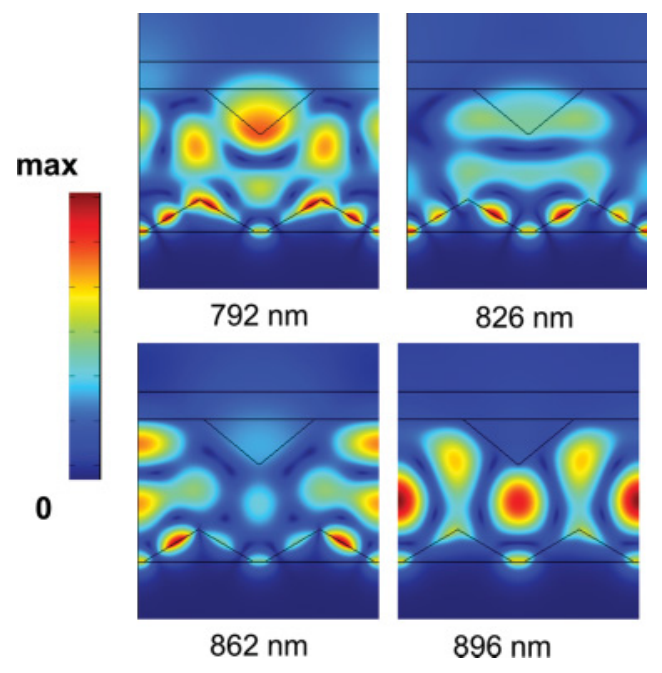

FIG. 7. (Color online) $\left|H_{z}\right|$ total field profiles at the resonances above $750 \mathrm{~nm}$ for the dual-periodicity structure in Fig. 6(a). 
In conclusion, we have shown that dual-periodic systems can provide better coupling efficiency to multiple waveguide and SPP modes, as compared to single-periodic systems. By using double periodicity, coupling efficiency to higherorder modes can be achieved without sacrificing coupling to lower-order modes, which can increase the overall absorption efficiency of the device.

To achieve solar cells with such multiperiod DIG structure, one can consider utilizing superstrate fabrication techniques. In superstrate cells, the solar cell structures are grown first on top of a transparent substrate. ${ }^{36}$ We can first pattern this substrate before depositing the solar cell materials on it. After deposition, we will have the top side available for additional patterning before applying the contacts.

\section{BLAZED DUAL-INTERFACE GRATINGS SYSTEMS}

Antisymmetric waveguide and SPP modes cannot be accessed by normal incident plane waves via symmetric grating systems. This problem can be bypassed by breaking the symmetry, for example, with blazed gratings which have asymmetry. Asymmetrical grating structures have been shown to be able to potentially give more absorption of normal incident light as they make dark modes accessible at normal incidence. $^{20,36}$ We compare the absorption spectrum of a symmetric DIG system [Fig. 8(a)] and its weakly blazed counterpart [Fig. 8(b)] in Fig. 8(c). The blazing is applied to

(a)

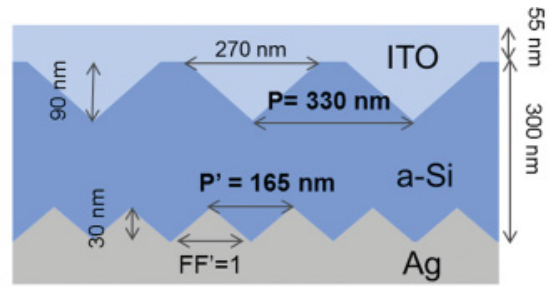

(b)

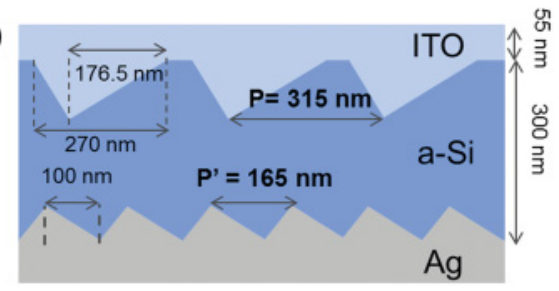

(c)

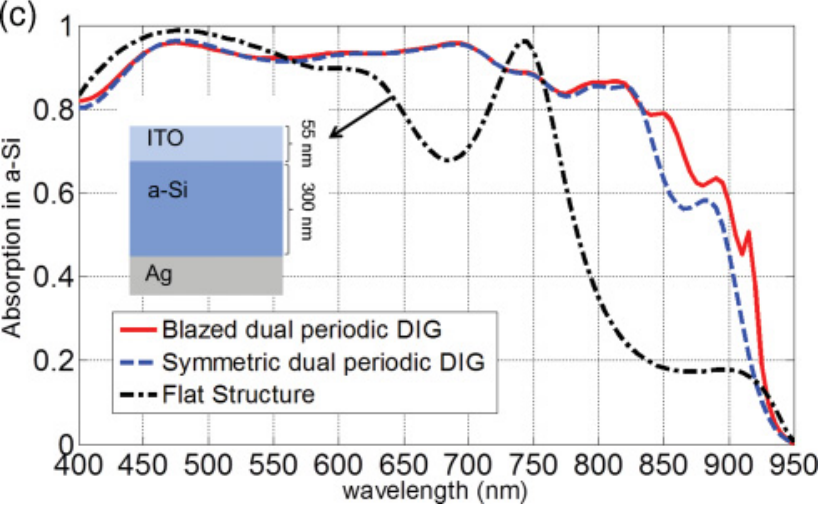

FIG. 8. (Color online) Structure schematic for (a) a symmetric DIG system and (b) when blazing is applied. (c) Absorption spectrum of structure (a) (dash line) and (b) (solid red line) for normal incidence compared with a planar reference (black dash-dot line). both the front and back grating. The absorption spectra show that weak blazing is sufficient to excite dark modes, as new peaks appear. Blazing changes both the scattering properties of the grating structure and the eigenmode field profile of these dark modes. Furthermore, we see that absorption at other wavelengths is preserved, particularly at the peaks already present for the symmetric structure. Dark modes are thus more sensitive to the blazing than bright modes. This implies that one can first optimize the symmetric grating to couple bright modes, e.g., with the period-doubling enhancements of Sec. IV, and then introduce blazing for further optimization.

We look into the details behind the additional resonances in the blazed system by examining and comparing their eigenmode field profiles in the symmetric and blazed cases (Fig. 9). The two new peaks at 850 and $915 \mathrm{~nm}$ correspond to a SPP and a dielectric mode, respectively. We can see [Fig. 9(a)] that there is only a weak distortion of the field profile due to the blazing for the waveguide mode, as compared to the SPP mode in Fig. 9(b). However, this minimum distortion in the eigenmode field profile [Fig. 9(a), left compared to right] is already enough to give a strong excitation because the change in the scattering properties of the gratings complement as mentioned before. The heavier distortion of the SPP dark mode [Fig. 9(b), left compared to right] is partially due to the fact that this mode is close to relatively broadband bright resonances, giving stronger interferences and a mixed modal profile. Although the SPP dark mode is uncoupled to other

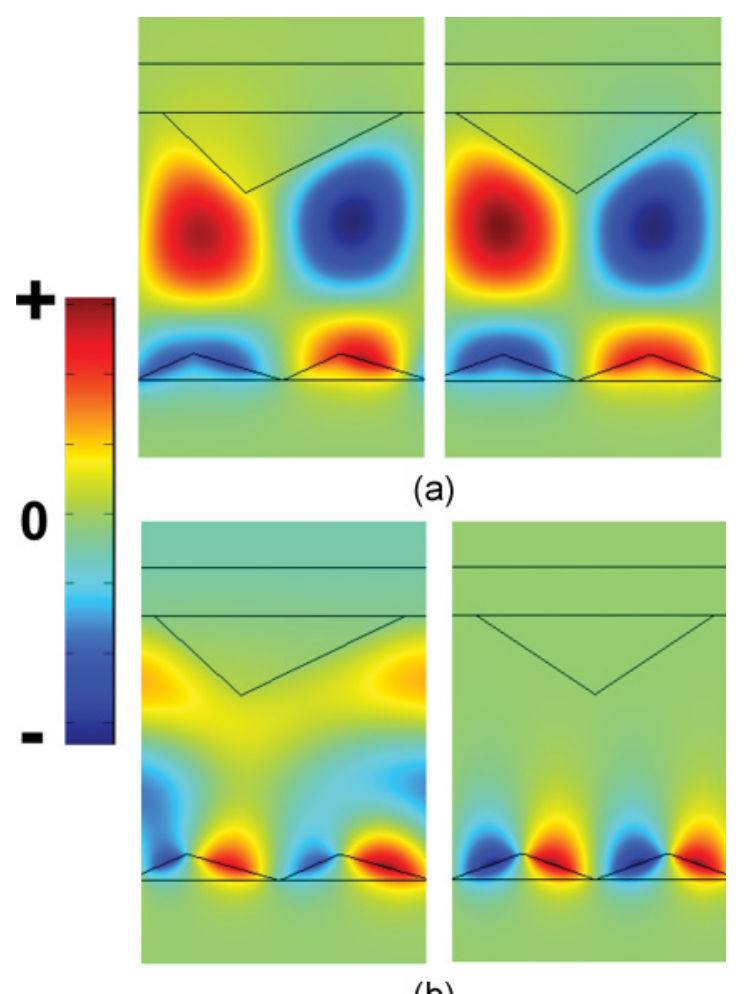

(b)

FIG. 9. (Color online) Dark modes $H_{z}$ profile in the symmetric DIG system and their accessible form in the blazed DIG system. (a) First-order waveguide mode in the two systems: blazed (left) and symmetric (right) at 915-nm wavelength. (b) SPP mode: blazed (left) and symmetric (right) at $850 \mathrm{~nm}$. 


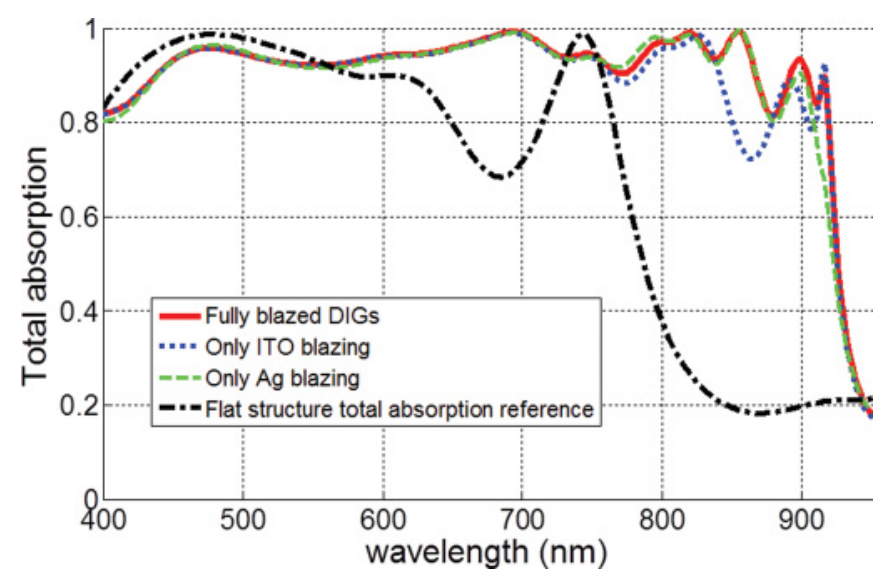

FIG. 10. (Color online) Total absorption spectra of a fully blazed DIG structure from Fig. 8(b) [solid red (gray) line] and of structures that have blazing on the ITO front grating only (dotted blue line) and on the Ag back grating only (dashed green line). For comparison, we show also the total absorption spectrum of a reference flat structure as in Fig. 8(c) (dash-dot black line).

resonances in the symmetric grating system, other bright modes can interfere with this previously "dark" SPP mode in the blazed system, since now this mode is also accessible for normal incident light.

Blazing both front and bottom gratings is important in achieving the desired result. Figure 10 shows the effect of blazing only one side of the DIG structure on the absorption spectrum as compared to blazing both. We show here the total absorption spectrum (including metal absorption) to give a more direct idea of the coupling efficiency. The resonances which correspond to previously dark modes give above $90 \%$ absorption, indicating that the coupling of incoming light to these modes is already strong with just a weak blazing. The dark waveguide mode resonance at $915 \mathrm{~nm}$ will not be excited if we only blaze the back grating, and the dark SPP mode resonance at $850 \mathrm{~nm}$ is not excited if we only blaze the ITO side. This again shows the power and flexibility of combining both front and back gratings. Tailoring of the scattering and eigenmode field profile to access dark modes can be accomplished with more degrees of freedom in a DIG structure.

To demonstrate that high coupling efficiency to multiple guided modes is possible in blazed dual-periodic DIG systems, we show an optimized system in Fig. 11 and compare with its nonblazed counterpart. In the blazed system [Fig. 11(b)] we apply different blazing directions for the top and bottom gratings. In addition, we shift the front grating slightly with respect to the lower grating, also leading to a more optimized absorption. The red dashed circle line in Fig. 11(c) shows nearly $100 \%$ total absorption at guided mode resonances at longer wavelengths (>700 nm). Thus, the blazed dual-periodic DIG structure can indeed support a strong coupling efficiency with more modes, leading to an integrated absorption efficiency in the a-Si layer for normal incidence of $87.36 \%$ for TM polarization. The flat structure reference (ITO $60 \mathrm{~nm} / \mathrm{a}-\mathrm{Si} 290 \mathrm{~nm} / \mathrm{Ag}$ ) in Fig. 11(c) gives a normal incidence integrated absorption efficiency of $73.97 \%$. Though we focus on optimizing the conditions for TM-polarized light, we can see in Fig. 11(c) (magenta dots) (a)

(b)
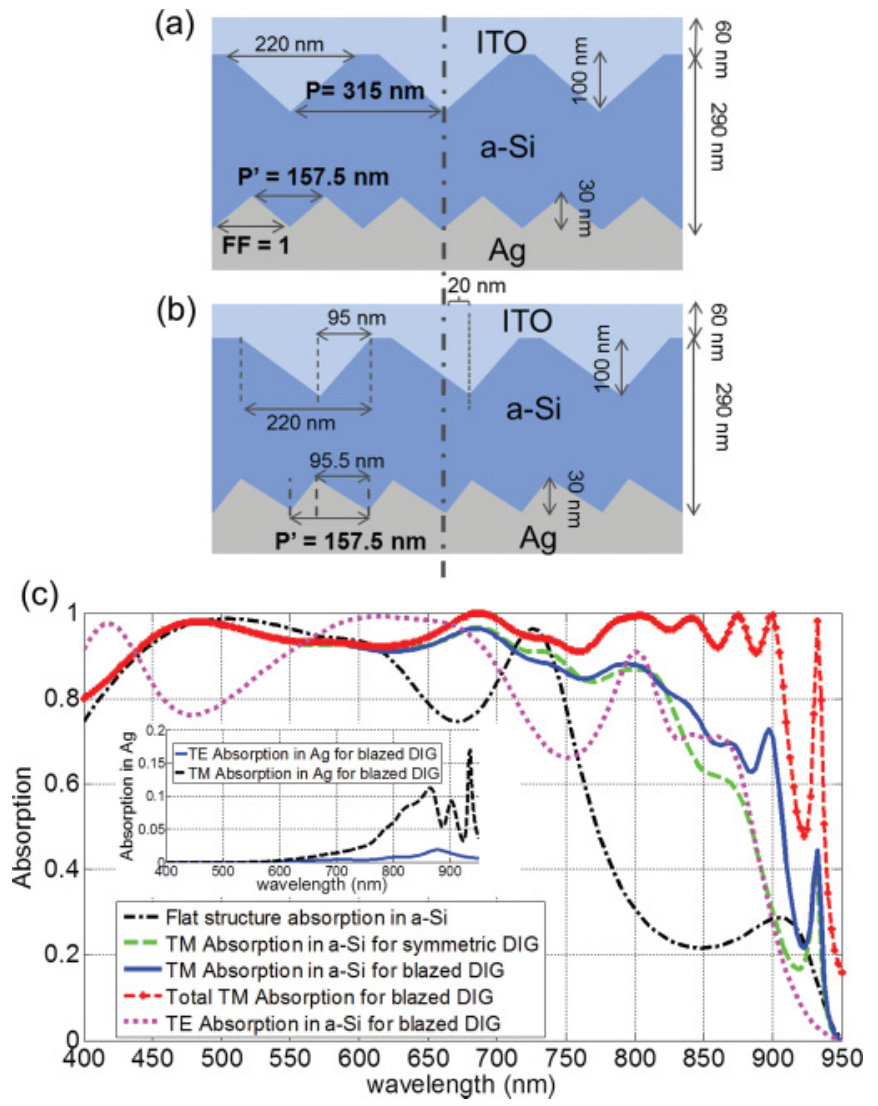

FIG. 11. (Color online) (a) Symmetric dual-periodic DIG system. (b) Optimized blazed dual-periodic DIG system providing high coupling efficiency to multiple guided modes. (c) Absorption spectra of the structures along with a planar reference (ITO 60 $\mathrm{nm} / \mathrm{a}-\mathrm{Si} 290 \mathrm{~nm} / \mathrm{Ag}$ ). Inset of (c) shows the absorption in the $\mathrm{Ag}$ back contact for (b).

that there is also improvement of TE-polarized light absorption, although in some wavelength ranges the absorption decreases. The integrated absorption efficiency for normal incident TE-polarized light is $81.53 \%$. For extra reference, we plot the absorption in the Ag back contact of the blazed DIG structure [Fig. 11(b)] for both polarizations in the inset of Fig. 11(c). We see in the inset that there is a small absorption in the $\mathrm{Ag}$ back contact, and we note that a significant part of the absorption can happen in the ITO layer at wavelengths $>900 \mathrm{~nm}$.

Figure 12 shows the integrated absorption efficiency $\left(A b s_{\text {int }}\right)$ (normalized to incoming power at normal incidence) as a function of incidence angle for the optimized DIG structures and the planar reference of Fig. 11(c). We see that the absorption of the blazed structure in Fig. 11(b) for both TE (black dashed) and TM (blue solid line) cases surpasses the flat reference absorption (green dash-dotted for TE and purple squares for TM) until $40^{\circ}$ angle of incidence. Beyond $40^{\circ}$, the TM case absorption in the blazed structure is still above the flat reference case although the TE case absorption in the blazed structure is less than the flat layer TM case absorption. Comparing $A b s_{\text {int }}$ for optimized symmetric (red dots) and blazed (blue solid)DIG structure, we see that the blazed structure improves the near-normal incidence absorption performance without much affecting the 


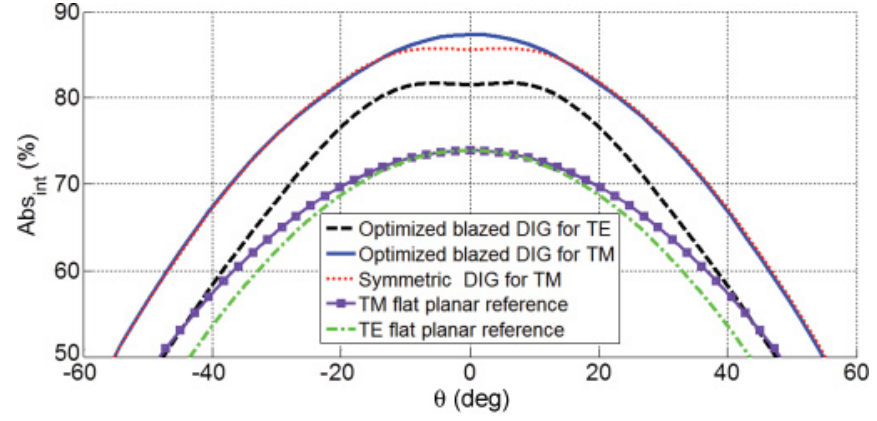

FIG. 12. (Color online) Integrated absorption efficiency vs incidence angle for the structures in Figs. 11(a) and 11(b) and a flat planar reference (ITO $60 \mathrm{~nm} / \mathrm{a}-\mathrm{Si} 290 \mathrm{~nm} / \mathrm{Ag}$ ).

absorption performance at larger angles. Also, because of the weak blazing, only a very weak dependence between the two orientations of $\theta(+\theta$ or $-\theta)$ for the blazed DIG structure is distinguished. Around normal incidence $\left( \pm 13.5^{\circ}\right)$, the difference between the two orientations of $\theta$ is only in the range of $0.1 \%$ while at large angles it can reach to $0.5 \%$, which is still quite weak. Though the difference of $A b s_{\text {int }}$ between the symmetric and blazed cases here is only $1.7 \%$ for normal incidence, the results show that weak blazing can allow coupling to more modes at normal incidence without ruining the angular performance at larger angles. More enhancement can be achieved in systems where the wavelength range window for absorption enhancement is larger.

\section{CONCLUSION}

We discussed the strategies of using dual-interface grating structures for absorption enhancement in thin-film solar cells. We propose the multiperiodic DIG structure for enhancing the coupling efficiency to both plasmonic and dielectric modes. These structures can give stronger coupling to higher-order guided modes, without sacrificing the coupling efficiency to lower-order modes. Weakly blazed grating structures give access to previously dark modes with very high coupling efficiency at normal incidence, without sacrificing the absorption at other wavelengths and at larger angles. Blazing of the front and back grating provides specific access to guided modes, e.g., blazing at the front dominantly influences the dielectric waveguide modes and not the SPP mode at the back contact. Combining blazing and dual periodicity in DIG structures leads to more accessible modes with strong coupling efficiency and hence achieves a broader absorption enhancement.

\section{ACKNOWLEDGMENTS}

This research was supported by the IWT Institute for the Promotion of Innovation by Science and Technology in Flanders via the SBO project "SilaSol," by the Interuniversity Attraction Poles program of the Belgian Science Policy Office under Grant No. IAP P6-10 "photonics@be" and by COST Action MP0702. *aimi.abass@elis.ugent.be

${ }^{1}$ K. L. Chopra, P. D. Paulson, and V. Dutta, Prog. Photovoltaics 12, 69 (2004).

${ }^{2}$ D. N. Weiss, H. C. Yuan, B. G. Lee, H. M. Branz, S. T. Meyers, A. Grenville, and D. A. Keszler, J. Vac. Sci. Technol. B 28, C6M98 (2010).

${ }^{3}$ B. Paäivaänranta, P. K. Sahoo, E. Tocce, V. Auzelyte, Y. Ekinci, H. H. Solak, C.-C. Liu, K. O. Stuen, P. F. Nealey, and C. David, ACS Nano 5, 1860 (2011).

${ }^{4}$ R. Tena-Zaera, M. A. Ryan, A. Katty, G. Hodes, S. Bastide, and C. Levy-Clement, C. R. Chim. 9, 717 (2006).

${ }^{5}$ V. E. Ferry, L. A. Sweatlock, D. Pacifici, and H. A. Atwater, Nano Lett. 8, 4391 (2008).

${ }^{6}$ F. J. Beck, S. Mokkapati, and K. R. Catchpole, Prog. Photovoltaics 18, 500 (2010).

${ }^{7}$ S. Fahr, T. Kirchartz, C. Rockstuhl, and F. Lederer, Opt. Express 19, A865 (2011).

${ }^{8}$ O. Isabella, M. Zeman, J. Krč, and M. Topič, in 25th European Photovoltaic Solar Energy Conference and Exhibition, Valencia, Spain, 2010 (WIP, Munich, 2010), p. 3273.

${ }^{9}$ S. Mokkapati, F. J. Beck, A. Polman, and K. R. Catchpole, Appl. Phys. Lett. 95, 053115 (2009).

${ }^{10}$ W. E. I. Sha, W. C. H. Choy, and W. C. Chew, Opt. Express 18, 5993 (2010).

${ }^{11}$ I. Gordon, L. Carnel, D. Van Gestel, G. Beaucarne, and J. Poortmans, Prog. Photovoltaics 15, 575 (2007).

${ }^{12}$ K. Q. Le, A. Abass, B. Maes, P. Bienstman, and A. Alù, Opt. Express 20, A39 (2012).
${ }^{13}$ H. Shen and B. Maes, Opt. Express 19, A1202 (2011).

${ }^{14}$ D. Madzharov, R. Dewan, and D. Knipp, Opt. Express 19, A95 (2011).

${ }^{15}$ A. Abass, H. Shen, P. Bienstman, and B. Maes, J. Appl. Phys. 109, 023111 (2011).

${ }^{16}$ C. J. Min, J. Li, G. Veronis, J. Y. Lee, S. H. Fan, and P. Peumans, Appl. Phys. Lett. 96, 133302 (2010).

${ }^{17}$ C. C. Chao, C. M. Wang, and J. Y. Chang, Opt. Express 18, 11763 (2010).

${ }^{18}$ V. E. Ferry, M. A. Verschuuren, H. B. T. Li, E. Verhagen, R. J. Walters, R. E. I. Schropp, H. A. Atwater, and A. Polman, Opt. Express 18, A237 (2010).

${ }^{19}$ J. Gjessing, E. S. Marstein, and A. Sudbø, Opt. Express 18, 5481 (2010).

${ }^{20}$ Z. Yu, A. Raman, and S. Fan, Opt. Express 18, A366 (2010).

${ }^{21}$ V. E. Ferry, A. Polman, and H. A. Atwater, ACS Nano 5, 10055 (2011).

${ }^{22}$ H. A. Atwater and A. Polman, Nat. Mater. 9, 205 (2010).

${ }^{23}$ K. R. Catchpole and A. Polman, Opt. Express 16, 21793 (2008).

${ }^{24}$ F. Hallermann, C. Rockstuhl, S. Fahr, G. Seifert, S. Wackerow, H. Graener, G. von Plessen, and F. Lederer, Phys. Status Solidi A 205, 2844 (2008).

${ }^{25}$ H. H. Shen, P. Bienstman, and B. Maes, J. Appl. Phys. 106, 073109 (2009).

${ }^{26}$ C. Rockstuhl, S. Fahr, and F. Lederer, J. Appl. Phys. 104, 123102 (2008). 
${ }^{27}$ E. B. Grann and M. G. Moharam, J. Opt. Soc. Am. A 13, 988 (1996).

${ }^{28}$ E. B. Grann, M. G. Varga, and D. A. Pommet, J. Opt. Soc. Am. A 12, 333 (1995).

${ }^{29}$ M. K. Kim, D. K. Yi, and U. Paik, Langmuir 26, 7552 (2010).

${ }^{30}$ P. B. Johnson and R. W. Christy, Phys. Rev. B 6, 4370 (1972).

${ }^{31}$ SOPRA-SA, [http://www.sopra-sa.com/].

${ }^{32}$ A. Yariv and P. Yeh, Photonics: Optical Electronics in Modern Communications (Oxford University Press, USA, 2006).
${ }^{33}$ J. D. Joannopoulos, S. G. Johnson, J. N. Winn, and R. D. Mead, Photonic Crystals: Molding the Flow of Light (Princeton University Press, Princeton, 2008).

${ }^{34}$ S. R. K. Rodriguez, A. Abass, B. Maes, O. T. A. Janssen, G. Vecchi, and J. Gómez Rivas, Phys. Rev. X 1, 021019 (2011).

${ }^{35}$ H. P. Zappe, Fundamentals of Micro-Optics (Cambridge University Press, New York, 2010).

${ }^{36}$ X. Sheng, S. G. Johnson, J. Michel, and L. C. Kimerling, Opt. Express 19, A841 (2011). 